\title{
Correlation of Progesterone Receptor B in Endometrial Tissue of Menstrual Blood in Patients with and without Endometriosis
}

\author{
${ }^{1}$ Ruswana Anwar, ${ }^{2}$ Amy I Soenggono, ${ }^{3}$ Zulvayanti, ${ }^{4}$ Tita H Madjid, ${ }^{5}$ Wiryawan Permadi, ${ }^{6}$ Jusuf S Effendi
}

\begin{abstract}
Background: Endometriosis is a progressive chronic gynecological disease. There are indications that endometriosis patients have lower progesterone receptor B (PR-B) levels. Menstrual blood contains the same mRNA derived from endometrium cells. The objective of this study was to analyze the different levels of PR-B in the menstrual blood of women with endometriosis compared to nonendometriosis.
\end{abstract}

Materials and methods: A case control study was conducted on 42 women administered to the Gynecology and Reproductive Endocrinology and Fertility Clinic FKUP/Dr Hasan Sadikin Hospital and its networks from December 2016 to January 2017. Through interviews, physical examination and investigation, patients were classified as those with endometriosis and nonendometriosis patients as controls. Surgery was performed to obtain lesion biopsy for endometriosis degree determination and storage. Quantitative RT-PCR for the PR-B mRNA in the menstrual blood samples was performed. Based on the histopathology results and diagnostic confirmation, differences in the PR-B levels in menstrual blood endometrium for endometriosis cases and non-endometriosis women were assessed. Differences in the PR-B levels between endometriosis patients with mild and severe degrees were also investigated.

Results: In patients with endometriosis, the mean and median levels of PR-B was significantly lower than non-endometriosis $(p<0.05)$. ROC analysis resulted in a cutoff value of $\leq 1.1355$ $\mu \mathrm{g} / \mathrm{dL}$ with $90.5 \%$ sensitivity, $81 \%$ specificity and $85.7 \%$ accuracy for prevalence odds ratio (POR) at 7.85. The PR-B levels strongly inversely correlated with the degree of endometriosis ( $r s=-0.817 ; p<0.001)$.

Conclusion: The levels of PR-B in eutopic endometrial tissue of menstrual blood in patients with endometriosis is lower than those in nonendometriosis women. A higher degree of endometriosis

\footnotetext{
${ }^{1-5}$ Researcher, ${ }^{6}$ Professor

${ }^{1-3}$ Department of Obstetric and Gynaecology, Padjadjaran University, Hasan Sadikin Hospital, Bandung, Indonesia

${ }^{4,5}$ Division of Fertility and Reproductive Endocrinology, Department of Obstetrics and Gynaecology, Padjadjaran University, Hasan Sadikin Hospital, Bandung, Indonesia

${ }^{6}$ Division of Fetomaternal Medicine, Department of Obstetrics and Gynaecology, Padjadjaran University, Hasan Sadikin Hospital, Bandung, Indonesia

Corresponding Author: Ruswana Anwar, Researcher, Department of Obstetric and Gynaecology, Padjadjaran University, Hasan Sadikin Hospital, Bandung, Indonesia, Phone: +62 811221414, +62 8112256868, e-mail: ruswana.anwar@ unpad.ac.id, ruswana_anwar@yahoo.com
}

is correlated with lower levels of PR-B in patients with endometriosis.

Keywords: Degree of endometriosis, Endometriosis, Menstrual blood, Progesterone receptor B, Quantitative real-time-polymerase chain reaction.

How to cite this article: Anwar R, Soenggono Al, Zulvayanti, Madjid TH, Permadi W, Effendi JS. Correlation of Progesterone Receptor B in Endometrial Tissue of Menstrual Blood in Patients with and without Endometriosis. J South Asian Feder Obst Gynae 2018;10(Suppl 1):310-315.

Source of support: Nil

Conflict of interest: None

Date of received: 19 February 2017

Date of acceptance: 2 May 2018

Date of publication: July 2019

\section{BACKGROUND}

Endometriosis is described as the presence of endometrial-like tissue outside of the uterine cavity, which is the underlying cause of chronic inflammation found in approximately $10 \%$ of women at reproductive age. ${ }^{1}$ Endometriosis is an important gynecological issue, because of its chronicity and its progressive impacts in decreasing quality of life as well as causing infertility, particularly for women at reproductive age. There are several stages of endometriosis, with stage I exhibiting minimum symptoms and stage IV that manifests in superficial as well as deep implants, which may include enlarged ovarian endometrioma, often accompanied by severe adhesion. ${ }^{2-4}$

Many hypotheses have been brought forward to explain the pathogenesis of endometriosis, such as retrograde menstruation, coelomic metaplasia, and hormonal induction theories, in addition to the roles of genetic factors and humoral or cellular immunity. ${ }^{2-9}$ However, none of these theories could fully explain the pathogenesis of endometriosis.

Eutopic and ectopic endometrium of endometriosis patients are different from those of nonendometriosis women in terms of its ability to locally produce estrogen and prostaglandin in high amount, and its resistance to progesterone. ${ }^{10}$ Several characteristics of endometrium tissue in endometriosis patients include (1) the high production of estrogen locally, (2) the ability to implant 
in peritoneum, (3) the tendency to proliferate and invade normal tissue, (4) highly aggressive, (5) resistance to physiological apoptosis, and (6) excessive angiogenesis. ${ }^{11}$

Endometriosis implant experience changes influenced by the estrogens a progesterone levels across the menstrual cycle, parallel with normal changes in the endometrium. Exposure to estrogen is the most dominant endocrine factor in its development and differentiation, while progesterone inhibits endometriosis implantation and is associated with disease regression. These are in accordance with the roles of estrogen in the proliferation phase and progesterone as its inhibitor in the secretory phase of the menstrual cycle. Hormonal influence on the development of endometriosis is also evident in the ability of steroid hormones to stimulate endometriosis, and disease regression after surgery as the effect of danazol, medroxyprogesterone acetate (MPA) and gonadotropin-releasing hormone (GnRH) analogs. $^{12}$

The inhibition of endometriosis implantation by progesterone is a paracrine action on endometrium stromal cells to increase the production of retinoic acid, which induces the expression and activation $17 \beta$-hydroxysteroid dehydrogenase (17 $\beta$-HSD) type 2 enzyme. ${ }^{13-15}$ This enzyme catalyzes the conversion of estriol into estrone that is biologically less active. Several studies have shown that endometriosis patients have lower expression of $17 \beta$-HSD type 2 compared to normal endometrium tissues. ${ }^{16-20}$ Furthermore, transcriptomic analysis on endometrium cells showed that eutopic endometrium of endometriosis patients exhibits a lower expression of progesterone-responsive genes, which may disrupt the transition of proliferative to secretory phases. ${ }^{21,22}$ This indicates that response to progesterone is one of the predisposition factors in the development of ectopic endometrial implant.

Progesterone effects are mediated by a progesterone receptor $(\mathrm{PR})$, which is grouped in the nuclear hormone receptor family. It has been stipulated that the progressiveness of endometriosis is associated with a decrease of PR expression. There are two main types of PR that have been identified: PR-A and PR-B. PR-A is a 94-kDa protein, while PR-B is larger at $114 \mathrm{kDa}$ with extra 164 amino acids in the N-terminal. These isoforms are the results of differential translation initiation at the alternative messenger RNA (mRNA) oral ternative transcription promoters. ${ }^{23-26}$ Previous studies have shown that PR-B holds an important role as the main effector in response to progesterone. ${ }^{27-29}$ In the majority of cell types, PR-B has strong transcription activities induced by its ligands, while PR-A has minimal transcription activities and may act as negative feedback to the activity of PR-B, depending on the relative ratio of PR-A:PR-B. It has also been reported that the disruption of the regulation in PR expression is found in endometriosis tissues. ${ }^{21,30}$

Studies on PR isoforms in endometriosis is contradictory at best. Some investigation reports that using assays that do not differentiate PR-A and PR-B, PR levels are lower in ectopic endometrium compared to eutopic tissue. However, other investigators reported no significant difference. Attia et al. used immunoblotting to detect PR-A and PR-B levels. They reported the absence of PR-B in peritoneal endometriosis tissue and lower-than-eutopic expression of PR-A. Ovarian endometriosis tissue showed a dominant expression of PR-A, with observation of hypermethylation of the PR-B promoter-consistent with the decrease of PR-B expression. ${ }^{22,31,32}$ Progesteroneplays an important role in the development of endometriosis, and further studies using specific and sensitive methods are warranted to investigate the levels of PR-A and PR-B, its post-translation modification forms, as well as its transcriptional activities in contributing to the role of PR in pathophysiology of endometriosis. ${ }^{33}$

Clinically useful biomarkers ideally have high specificity and sensitivity. In addition, it is preferred to develop diagnostic assays that utilize specimens obtained through non-invasive or minimally invasive procedures. ${ }^{34}$ Menstrual blood from the first or second day of menstrual cycle contains the same DNA and mRNA as those originated from the endometrium, as reported by Fiegl et al. ${ }^{35}$ Other investigators also reported that there is no significant difference in gene polymorphism between endometriosis or non-endometriosis patients using analysis of menstrual blood. ${ }^{36}$ The objective of this study is to use data obtained from menstrual blood, to quantify the levels of PR-B in endometriosis patients compared to nonendometriosis women, and its correlation with the severity of endometriosis. The results of this study are expected to broaden the clinical and molecular understanding of the roles of progesterone in the pathophysiology of endometriosis.

\section{MATERIALS AND METHODS}

\section{Study Subjects}

Samples used in this study were at least $1 \mathrm{~mL}$ of whole blood EDTA samples from menstrual bleeding day 1 or day 2 from patients aged 18-45 years old that were enrolled for cystectomy or salpingo-ovariectomy procedures, either by laparoscopy or laparotomy, in RS Dr Hasan Sadikin and its affiliations within the time frame (December 2016-January 2017). Samples of endometriosis lesions obtained during the procedure were preserved in $10 \%$ formalin for further histopathology analysis to determine the stage of endometriosis in cases or status of nonendometriosis in controls. All patients were required to have regular menstrual cycles for at least 3 months 
before the procedure and had not received any hormonal therapy during those 3 months. None of the patients were diagnosed with chronic inflammation, infection, or other gynecological malignancies. For case samples, endometriosis stage was determined based on classifications as defined by the American Society for Reproductive Medicine (ASRM), based on laparoscopy findings. ${ }^{2-4}$ A total of 21 case control pairs was obtained. Individual informed consent was obtained from each participant. This study protocol was in accordance to and approved by the ethical committee of RS Hasan Sadikin (LB.02.01/ C02/1338/I/2017).

\section{RNA Extraction and cDNA Synthesis}

Menstrual blood samples were processed using SV total RNA isolation system kit (Promega, Madison, WI, USA) as described by the manufacturer. RNA solution was quantified using spectrophotometer at 260, 280 and 320 $\mathrm{nm}$ to determine the quantity and quality of RNA. cDNA synthesis was performed using iScriptc DNA synthesis kit (Biorad, Hercules, CA, USA). The reaction mixture was incubated at $25^{\circ} \mathrm{C}$ for 5 minutes, followed by $42^{\circ} \mathrm{C}$ for 30 minutes, and $85^{\circ} \mathrm{C}$ for 5 minutes.

\section{Quantitation of mRNA Levels for the Expression of PR-B}

The levels of mRNA expression were determined using relative quantitative real time-PCR (qRT-PCR) using GAPDH as the reference gene. Primers used in this study were described in Table 1. The qRT-PCR reaction was performed using SsoFast ${ }^{\mathrm{TM}}$ EvaGreen ${ }^{\circledR}$ Supermix (Biorad, Hercules, CA, USA) with preincubation at $95^{\circ} \mathrm{C}$ for 3 minutes, followed by 39 cycles of denaturation at $95^{\circ} \mathrm{C}$ for 15 seconds, annealing at $57^{\circ} \mathrm{C}$ for 20 seconds, and extension at $72^{\circ} \mathrm{C}$ for 25 seconds in Duplo. Analysis of gene expression levels was done using the comparative CT method and standard curve to quantify the mRNA in each sample.

\section{Statistical Analysis}

Data were analyzed using the SPSS ver. 21.0 software for Windows. ${ }^{27}$ For numerical data, Sapiro-Wilk test was performed to check data normality. Mann-Whitney test was utilized to analyze non-normally distributed data. Kolmogorov-Smirnov test was used to analyze categorical data. To determine the cutoff value for progesterone

Table 1: Primers used in this study

\begin{tabular}{lll}
\hline No & Primer & Nucleotides $\left(5^{\prime} \rightarrow 3^{\prime}\right)$ \\
\hline 1 & GAPDH-F & GAA GGT GAA GGT CGG AGT C \\
2 & GAPDH-R & GAA GAT GGT GAT GGG ATT TC \\
3 & PRB-F & TAG TGA GGG GGC AGT GGA AC \\
4 & PRB-R & AGG AGG GGG TTT CGG GAA TA \\
\hline
\end{tabular}

levels as a predictor for endometriosis, analysis using receiver operating characteristics (ROC) curve was used. Spearman correlation test was performed to analyze the correlation between PR levels and endometriosis severity. Significance was determined at a $p$ value of $\leq 0.05$.

\section{RESULTS}

\section{Sample Characteristics}

Out of 42 patients, 21 had endometriosis (35.6 years old \pm 4.9 years), and 21 were non-endometriosis (33.7 years old \pm 5.4 years). No significant difference was observed between the two groups for age and body mass index (BMI) distributions (Table 2).

\section{Comparison of mRNA Levels for PR-B Protein Expression}

Mean and median values of progesterone levels were significantly lower in endometriosis compared to nonendometriosis group ( $p$ value $<0.05$; Table 3 ).

Calculation of cutoff value for progesterone levels that could be used to predict endometriosis risk using ROC curve showed a value at $\leq 1.1355 \mu \mathrm{g} / \mathrm{dL}$ with $90.5 \%$ sensitivity, $81 \%$ specificity, and $85.7 \%$ accuracy (Graph 1 ).

With cutoff value at $1.1355 \mu \mathrm{g} / \mathrm{dL}$, it was obtained that there was a 7.85 times higher risk in prevalence odds ratio (POR) analysis for the occurrence of endometriosis in women at reproductive age that had progesterone levels at $\leq 1.1355 \mu \mathrm{g} / \mathrm{dL}(p=0.001$; Table 4$)$.

Based on the severity of the disease, Spearman rank test was used to analyze the correlation between disease stage and progesterone levels. It was shown that there was a significantly strong negative correlation between PR-B levels and endometriosis severity $\left(\mathrm{r}_{\mathrm{s}}=-0.817 ; p<0.001\right)$ (Table 5 and Graph 2).

Table 2: Baseline characteristics of study subjects

\begin{tabular}{llll}
\hline & \multicolumn{2}{c}{ Group } & \\
\cline { 2 - 3 } Characteristics & $\begin{array}{l}\text { Endometriosis } \\
(n=21)\end{array}$ & $\begin{array}{l}\text { Nonendometriosis } \\
(n=21)\end{array}$ & $p$ value \\
\hline Age (years) & & & 0.228 \\
Mean (SD) & $35.6(4.9)$ & $33.7(5.4)$ & \\
Range & $26-43$ & $24-43$ & \\
& & & 0.209 \\
BMl $\left(\mathrm{kg} / \mathrm{m}^{2}\right)$ & & & \\
Mean $(\mathrm{SD})$ & $26.2(3.9)$ & $24.6(3.9)$ & $18.2-34.1$ \\
Range & $18.9-32.8$ & 18.2 & \\
\hline
\end{tabular}

Table 3: Comparison of PR-B levels among the two study populations

Groups

\begin{tabular}{llll}
$\begin{array}{l}\text { Levels of } P R-B \\
(\mu g / d L)\end{array}$ & $\begin{array}{l}\text { Endometriosis } \\
(n=21)\end{array}$ & $\begin{array}{l}\text { Nonendometriosis } \\
(n=21)\end{array}$ & $p$ value \\
\hline Mean $(\mathrm{SD})$ & $0.826(0.284)$ & $1.258(0.391)$ & $p<0.05$ \\
Median & 0.765 & 1.385 & \\
Range & $0.353-1.403$ & $0.359-1.756$ & \\
\hline
\end{tabular}




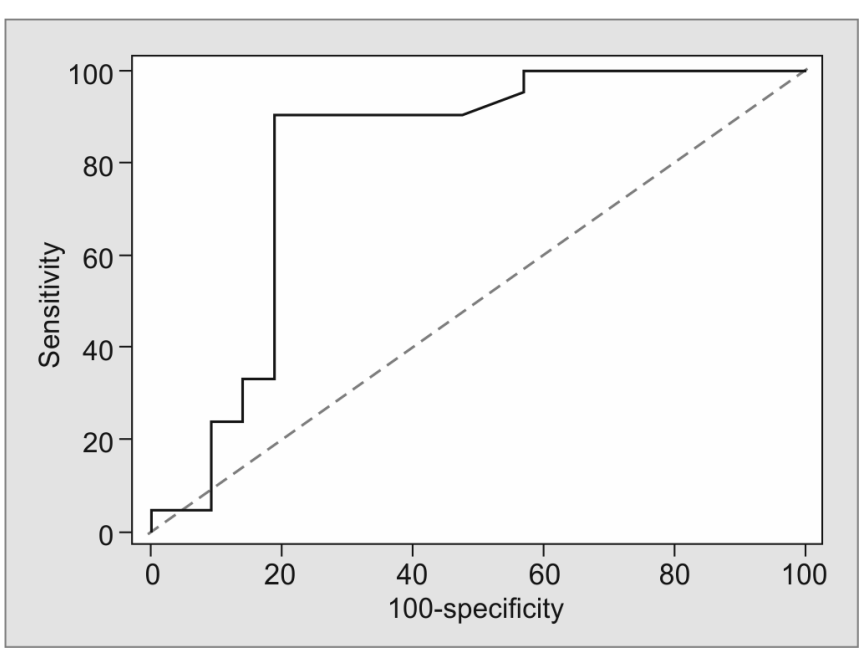

Graph 1: ROC curve to determine the cutoff value of PR-B levels as a predictor of endometriosis

Table 5: Correlation analysis of PR-B levels and severity of endometriosis

\begin{tabular}{|c|c|c|c|}
\hline \multirow[b]{2}{*}{ Variable } & \multicolumn{2}{|c|}{ Grade } & \multirow[b]{2}{*}{$p$ value } \\
\hline & $\begin{array}{l}I-I I \\
(n=7)\end{array}$ & $\begin{array}{l}\text { III-IV } \\
(n=14)\end{array}$ & \\
\hline PR-B levels & $1.156(0.168)$ & $0.6001(0.153)$ & $<0.001$ \\
\hline Mean (SD) & 1.073 & 0.704 & \\
\hline Median & $1.005-1.403$ & $0.353-0.866$ & \\
\hline Range & & & \\
\hline
\end{tabular}

\section{DISCUSSION}

Pathogenicity of endometriosis is still a topic of controversy, even with numerous genomic and proteomic studies on the roles of genetic factors, environmental influence, immunology, angiogenic and endocrine process. Meanwhile, diagnosis of endometriosis is also still ineffective, due to the various degree of clinical manifestations and the invasiveness of laparoscopy-biopsy procedure required as the gold standard of diagnostics. Noninvasive diagnostic techniques are currently under developments, including USG, CT scan, and MRI. However, the sensitivity and specificity of these methods are not ideal and only useful for advanced endometriosis. As a result, many cases are missed in the initial phase and only observed at later stages with more severe complications.

This study utilized endometrium samples found in menstrual blood of endometriosis and nonendometriosis women. This method was chosen due to its noninvasiveness, in comparison to prior investigations using biopsy samples of endometrium samples. Madjid et al. reported that endometrium cells in menstrual blood could represent eutopic endometrium cells. Therefore, these specimens could be used to investigate the levels of PR-B expression in endometrium tissue. ${ }^{36,37}$

The objective of this study was to investigate the difference of PR-B levels in endometriosis patients compared to non-endometriosis women, using day 2 menstrual blood specimens.
Table 4: Odds ratio analysis of PR-B levels at the cutoff value with the occurrence of endometriosis

\begin{tabular}{llll}
\hline \multirow{2}{*}{$\begin{array}{l}\text { Cutoff the value } \\
\text { of } P R-B(\mu \mathrm{g} / \mathrm{dL})\end{array}$} & $\begin{array}{l}\text { Gndometriosis } \\
(n=21)\end{array}$ & $\begin{array}{l}\text { Nonendometriosis } \\
(n=21)\end{array}$ & \\
\cline { 2 - 3 }$\leq 1.1355$ & $19(90.5 \%)$ & $4(19 \%)$ & 0.001 \\
$>1.1355$ & $2(9.5 \%)$ & $17(81 \%)$ & \\
\hline
\end{tabular}

POR (CI 95\%): 7.85 (2.09-29.50)

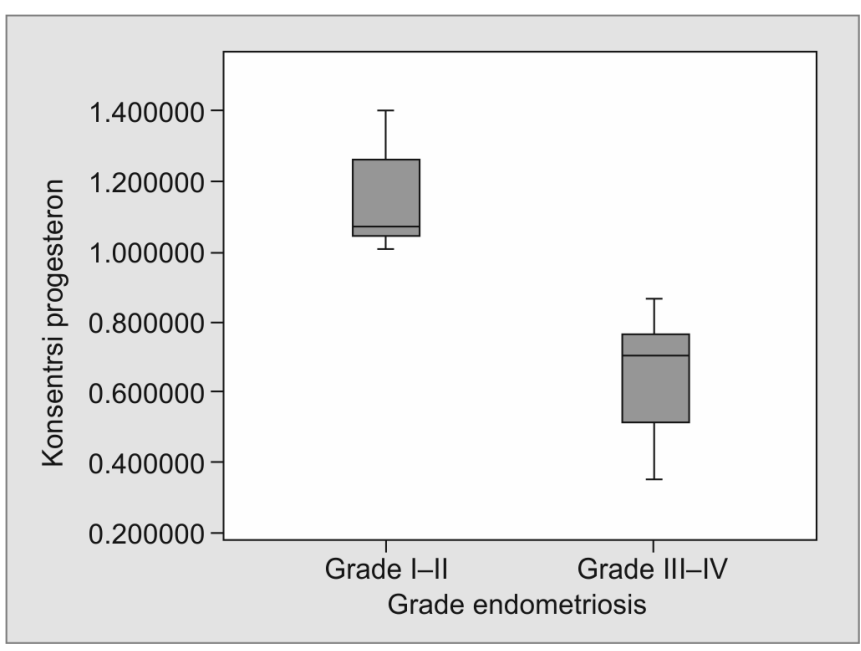

Graph 2: Correlation between PR-B levels and severity of endometriosis

Furthermore, the correlation between PR-B levels and severity of endometriosis was also analyzed. It was observed that there was no significant difference in the baseline characteristics of the two subject groups in this study. This finding was similar to reports by Ioannis et al. on a study of 535 endometriosis patients and 200 non-endometriosis infertile women that underwent laparoscopy or laparotomy procedures. They reported that endometriosis was not affected by age, height, body weight, IUD implantation, and smoking or caffeine status. BMI in endometriosis women tended to be lower than controls, but insignificant statistically. ${ }^{38}$

Eutopic endometrium in endometriosis women has underlying difference compared to the endometrium of non-endometriosis women. These differences include the variability of structure anomalies, the proliferation of immune components, adhesion molecules, proteolytic enzymes, and their inhibitors, steroid response and cytokine production, as well as gene expression dan protein production. ${ }^{39,40}$ These differences result in the implantation and development of endometriosis in the peritoneal cavity during retrograde menstruation.

Attia et al. showed that there was a disturbance in PR expression and regulation in the eutopic endometrium of endometriosis women. ${ }^{21,22,30}$ This fact was also observed in this study, with significantly higher PR-B levels in the non-endometriosis group compared to endometriosis patients. Mean value of PR-B levels in endometriosis 
patients were $0.765 \mu \mathrm{g} / \mathrm{dL}$, while it was $1.385 \mu \mathrm{g} / \mathrm{dL}$ for the nonendometriosis group.

Progesterone and progestin have long been used as therapeutic agents for endometriosis as analgetics, by mimicking pregnancy, to reduce estrogen biosynthesis by the ovaries and inhibit the proliferation and inflammation of endometriosis. Unfortunately, progestin action is shortlived, with up to $20-50 \%$ of endometriosis patients being non-responsive to progestin. This progesterone resistance in eutopic an ectopic endometrium of endometriosis women has been recorded in several investigations. In several endometriosis patients exhibiting pelvic pains, progestin therapy shows an unsatisfactory response. Molecular changes affected by progesterone on eutopic endometrium of endometriosis women are ineffective or undetectable. These in vivo observations indicate resistance in endometriosis that is more likely to be related to the decrease of PR levels in general and the lack of PR-B isoform in particular. ${ }^{2} \mathrm{PR}-\mathrm{B}$ is a strong gene activator, while PR-A acts as a negative feedback of PR-B actions when both proteins coexist in cell cultures. ${ }^{41}$

Iruela-Arispe et al. identified PR in endometrium endothelial cells and found that progesterone inhibited cell proliferation as induced by VEGF. In the secretory phase of the menstrual cycle, there is a marked increase of point density of blood vessels by angiogenesis.

Further confirmations are needed to determine the mechanisms and exact pathways that are affected by progesterone to induce angiogenesis of endometrium, including the interactions of PR-A and / or PR-B in endometrial endothelial cells. ${ }^{41}$

Studies on PR isoforms in endometriosis resulted in contradictory findings. Reports based on assays that in differentiate PR-A and PR-B showed lower PR levels in eutopic endometrium of endometriosis patients compared to normal women. However, other investigators reported no significant difference between the two groups. Attia et al. quantified PR-A and PR-B levels separately using immunoblotting methods and reported that peritoneal endometriosis lacks PR-B expression and has lower PR-A levels compared to eutopic endometrium tissue. ${ }^{21,22,30}$ On the other hand, mRNA analysis of ovarian endometriosis tissue showed a dominant expression of PR-A, with observed hypermethylation of PR-B promoter that explained the decrease of PR-B expression. ${ }^{22,31}$ In this study, it was indicated that PR-B mRNA levels could be analyzed from menstrual blood, and its expression levels were lower in endometriosis patients compared to nonendometriosis women.

As observed in this study, a cut off value of $\leq 1.1355$ $\mu \mathrm{g} / \mathrm{dL}$ in PR-B levels could be used to predict endometriosis using ROC curve analysis. With a $90.5 \%$ sensitivity, $81 \%$ specificity and $85.7 \%$ accuracy, this value showed
7.85 times higher odds ratio for endometriosis in women at reproductive age.

Diagnosis of endometriosis is obtained after histopathology examination on biopsy samples acquired in surgery. Endometriosis patients could be classified into four stages of advancement. For the purpose of this study, endometriosis ASRM grades 1 and 2 were grouped as mildly severe endometriosis while endometriosis ASRM grades 3 and 4 were classified as severe endometriosis. Kumar et al. reported that PR-A and PR-B expression levels in endometrial carcinoma were down-regulated, selectively for PR-B. This was correlated with the lack of response to progesterone therapy and worse differentiated carcinoma. ${ }^{42}$ Other studies on PR-A and PR-B levels in eutopic endometrium of endometriosis and nonendometriosis women showed similar observations.

It was observed in the study subjects that PR-B levels decreased in accordance with the severity of endometriosis. A significantly strong negative correlation was found between PR-Blevels with endometriosis severity $\left(r_{s}=-0,817\right.$; $p$ value $<0,001)$. In endometriosis grades 3 and 4 , worse inflammation response was noted. This indicated that a higher response of inflammation could be associated with lower PR levels, especially PR-B isoform.

There were some limitations in this study such as the absence of quantification for PR-A levels, estrogen- $\alpha$ receptor, estrogen- $\beta$ receptor, as well as other parameters using qRT-PCR to explain further the etiopathogenesis of endometriosis.

\section{CONCLUSION}

In conclusion, this study showed that PR-B levels in eutopic endometrium cells in menstrual blood specimens of endometriosis patients were significantly lower than nonendometriosis women. Furthermore, it was observed that women at reproductive age with progesterone levels $\leq 1,1355 \mu \mathrm{g} / \mathrm{dL}$ had 7.85 times higher risk of endometriosis. This cutoff value had $90.5 \%$ sensitivity, $81 \%$ specificity, and $85.7 \%$ accuracy. Interestingly, it was observed that advanced endometriosis was associated with lower PR-B levels in the menstrual blood of endometriosis patients. The use of menstrual blood in predicting the risk of endometriosis with the qRT-PCR method is a promising non-invasive diagnostic assay. Further investigations are required to understand the etiopathogenesis of endometriosis and to develop sensitive diagnostic modalities that are noninvasive by use of biomarkers showing significant changes in the eutopic endometrium tissues as obtained in a menstrual blood specimen.

\section{REFERENCES}

1. Hediger ML, Hartnett HJ, et al. Association of endometriosis with body size and figure. Feril Steril 2005;84(5):1366-1374. 
2. Bulun SE. Endometriosis. N Engl J Med 2009;360(3):268-279.

3. de Ziegler D, Borghese B, et al. Endometriosis and infertility: pathophysiology and management. Lancet (London, England) 2010;376(9742):730-738.

4. Kennedy S, Bergqvist A, et al. ESHRE guideline for the diagnosis and treatment of endometriosis. Hum Reprod 2005;20(10):26982704.

5. Leyland N, Casper R, et al. Endometriosis: diagnosis and management. JOGC 2010;32(7 Suppl 2):S1-32.

6. Shinohara A, Kutsukake M, et al. Protease-activated receptorstimulated interleukin-6 expression in endometriosis-like lesions in an experimental mouse model of endometriosis. J Pharm Sci 2012;119(1):40-51.

7. Ponce C, Torres M, et al. Nuclear factor kappaB pathway and interleukin- 6 are affected in eutopic endometrium of women with endometriosis. Reproduction (Cambridge, England) 2009;137(4):727-737.

8. Bergqvist A, Bruse C, et al. Interleukin $1 \beta$, interleukin-6, and tumor necrosis factor- $\alpha$ in endometriotic tissue and in endometrium. Feril Steril. 2001;75(3):489-495

9. A B. Endometriosis. A B, editor. Jakarta: Media Aesculapius FakultasKedokteranUniversitas Indonesia; 2008. p. 1-31.

10. Speroff L, Fritz MA. Clinical gynecologic endocrinology and infertility: lippincott Williams \& Wilkins; 2005.

11. Vinatier D, Cosson $M$, et al. Is endometriosis an endometrial disease? Eur J Obstet Gynecol Reprod Biol 2000;91(2):113-125.

12. Lessey BA, Metzger DA, et al. Immunohistochemical analysis of estrogen and progesterone receptors in endometriosis: comparison with normal endometrium during the menstrual cycle and the effect of medical therapy. Feril Steril 1989;51(3):409-415.

13. Casey M, MacDonald P, et al. 17 beta-Hydroxysteroid dehydrogenase type 2: chromosomal assignment and progestin regulation of gene expression in human endometrium. J Clin Invest 1994;94(5):2135.

14. Mustonen MV, Isomaa VV, et al. Human $17 \beta$-hydroxysteroid dehydrogenase type 2 Messenger ribonucleic acid expression and localization in term placenta and in endometrium during the menstrual cycle 1. J Clin Endocrinol Metab 1998;83(4):1319-1324.

15. Cheng Y-H, Yin P, et al. Retinoic acid (RA) regulates $17 \beta$-hydroxysteroid dehydrogenase type 2 expression in end metrium: interaction of RA receptors with specificity protein (SP) 1/SP3 for estradiol metabolism. JClin Endocrinol Metabb 2008;93(5):1915-1923.

16. Jiang J, Wu R-f, et al. Effect of mifepristone on estrogen and progesterone receptors in human endometrial and endometriotic cells in vitro.Fertil Sterill 2002;77(5):995-1000.

17. Halme J, Toma S, et al. A case of severe ovarian hyperstimulation in a healthy oocyte donor. Fertil Sterill 1995;64(4):857-859.

18. Kaunitz AM. Injectable depot medroxyprogesterone acetate contraception: an update for US clinicians. Int J Fertil Womens Med 1997;43(2):73-83.

19. Cheng Y-H, Imir A, et al. Stromal cells of endometriosis fail to produce paracrine factors that induce epithelial $17 \beta$-hydroxysteroid dehydrogenase type 2 gene and its transcriptional regulator Sp1: a mechanism for defective estradiol metabolism. Am J Obstett Gynecoll 2007;196(4):391. e1-91. e8.

20. Zeitoun K, Takayama K, et al. Deficient $17 \beta$-Hydroxysteroid Dehydrogenase Type 2 Expression in Endometriosis: Failure to Metabolize 17 $\beta$-Estradiol 1. J Clin Endocrinol Metab. 1998;83(12):4474-4480.

21. Burney RO, Talbi S, et al. Gene expression analysis of endometrium reveals progesterone resistance and candidate susceptibility genes in women with endometriosis. Endocrinology. 2007;148(8):3814-3826.
22. Younis A, Hawkins $K$, et al. Serum tumor necrosis factor- $a$, interleukin-6, monocyte chemotactic protein-1 and paraoxonase- 1 profiles in women with endometriosis,PCOSs, or unexplained infertility. J Assist Reprod Genet 2014;31(11):1445-1451.

23. Lebovic DI, Mueller MD, et al. Immunobiology of endometriosis. Feri 1Steril 2001;75(1):1-10.

24. Kastner P, Krust A, et al. Two distinct estrogen-regulated promoters generate transcripts encoding the two functionally different human progesterone receptor forms $\mathrm{A}$ and $\mathrm{B}$. EMBO J 1990;9(5):1603.

25. Sartorius CA, Melville MY, et al. A third transactivation function (AF3) of human progesterone receptors located in the unique N-terminal segment of the B-isoform. Mol Endocrinol 1994;8(10):1347-1360.

26. Leonhardt SA, Boonyaratanakornkit V, et al. Progesterone receptor transcription and non-transcription signaling mechanisms. Steroids 2003;68(10):761-770.

27. Evans RM. The steroid and thyroid hormone receptor superfamily. Science 1988;240(4854):889-895.

28. McEwan IJ. Nuclear receptors: one big family. Methods Mol Biol 2009:3-18.

29. Escriva H, Bertrand S, et al. The evolution of the nuclear receptor superfamily. Essays Biochem 2004;40:11-26.

30. Kao L, Germeyer A, et al. Expression profiling of endometrium from women with endometriosis reveals candidate genes for disease-based implantation failure and infertility. Endocrinology 2003;144(7):2870-2881.

31. Attia GR, Zeitoun K, et al. Progesterone receptor isoform a but not $b$ is expressed in endometriosis 1. JClin Endocrinol Metabb 2000;85(8):2897-2902.

32. Misao R, Iwagaki S, et al. Dominant expression of progesterone receptor form B mRNA in ovarian endometriosis. Horm Res 1999;52(1):30-34.

33. Patel B, Elguero S, et al. Role of nuclear progesterone receptor isoforms in uterine pathophysiology. Hum Reprod 2014:dmu056.

34. Guo S-W. Epigenetics of endometriosis. Mol Hum Reprod 2009;15(10):587-607.

35. R R. Hubungankadar estradiol zalir peritoneum, tampilan mRNA Reseptorprogesteron-B dan mRNA DNA metiltransferase-1 sertatingkatmetilasipengajureseptorprogesteron-B Endometrium dengankejadian endometriosis 2014:1-112.

36. Madjid T, Hernowo B, et al. The relationship of caspase3, caspase-9, MMP-9 expression and c-1562t mmp-9 gene polymorphism in menstrual blood as the etiopathogenesis marker to clinical endometriosis manifestation. Feri 1Steril 2015;3(104):e166.

37. Leyendecker G, Herbertz M, et al. Endometriosis results from the dislocation of basal endometrium. Hum reprod (Oxford, England) 2002;17(10):2725-2736.

38. Matalliotakis IM, Cakmak H, et al. Epidemiological characteristics in women with and without endometriosis in the Yale series. Arch Gynecol Obstet 2008;277(5):389-393.

39. Kitawaki J, Kusuki I, et al. Detection of aromatase cytochrome P-450 in endometrial biopsy specimens as a diagnostic test for endometriosis. Feri 1Steril 1999;72(6):1100-1106.

40. Sharpe Timms KL. Endometrial anomalies in women with endometriosis. Ann N Y Acad Sci 2001;943(1):131-147.

41. Girling JE, Rogers PA. Recent advances in endometrial angiogenesis research. Angiogenesis. 2005;8(2):89-99.

42. Kumar NS, Richer J, et al. Selective down-regulation of progesterone receptor isoform $\mathrm{B}$ in poorly differentiated human endometrial cancer cells: implications for unopposed estrogen action. Cancer Res 1998;58(9):1860-1865. 